\title{
Gradhiva
}

GRADHIV

Revue d'anthropologie et d'histoire des arts

11 | 2010

Grands hommes vus d'en bas

\section{Christine Laurière, Paul Rivet, le savant et le politique}

Paris, Publications scientifiques du Muséum national d'histoire naturelle, coll. " Archives », 2008, 723 p., illustrations

\section{Nélia Dias}

\section{(2) OpenEdition}

12 Journals

Édition électronique

URL : http://journals.openedition.org/gradhiva/1779

DOI : $10.4000 /$ gradhiva. 1779

ISSN : 1760-849X

Éditeur

Musée du quai Branly Jacques Chirac

Édition imprimée

Date de publication : 19 mai 2010

Pagination : 232-234

ISBN : 978-2-35744-025-8

ISSN : 0764-8928

Référence électronique

Nélia Dias, "Christine Laurière, Paul Rivet, le savant et le politique », Gradhiva [En ligne], 11 | 2010, mis en ligne le 09 mai 2010, consulté le 21 septembre 2020. URL : http://journals.openedition.org/gradhiva/ 1779 ; DOI : https://doi.org/10.4000/gradhiva.1779

Ce document a été généré automatiquement le 21 septembre 2020.

(c) musée du quai Branly 


\section{Christine Laurière, Paul Rivet, le savant et le politique}

Paris, Publications scientifiques du Muséum national d'histoire naturelle, coll. « Archives », 2008, 723 p., illustrations

Nélia Dias

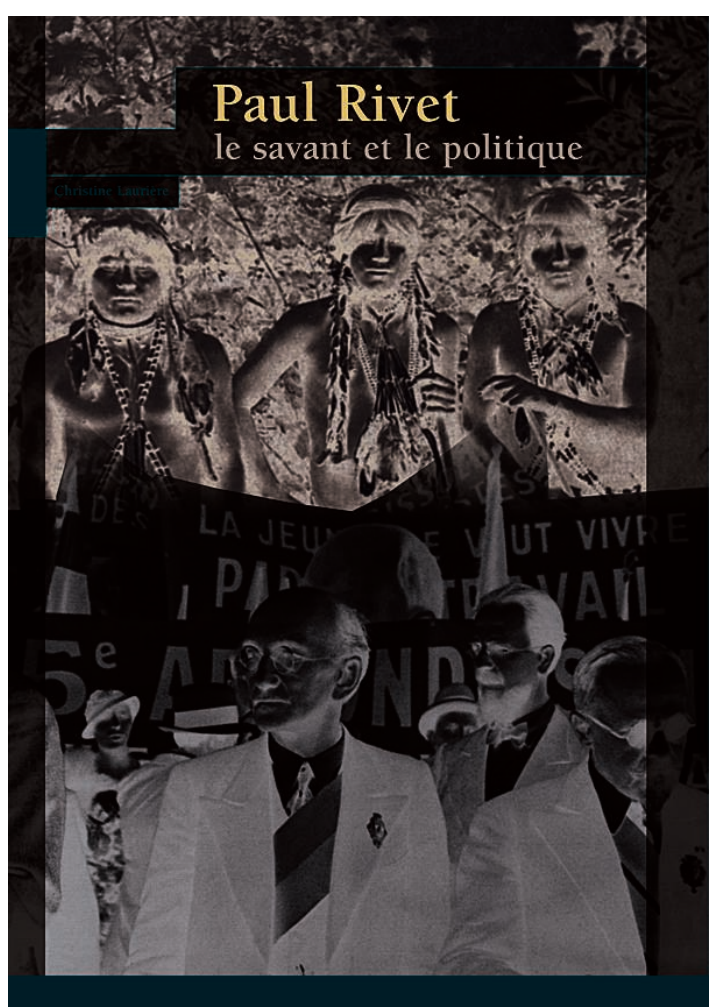

Voici enfin livrée au public la remarquable biographie intellectuelle de Paul Rivet (1876-1958) par Christine Laurière. Ce travail, issu d'une thèse de doctorat soutenue à l'École des hautes études en sciences sociales en 2006, fera date tant par l'extraordinaire érudition de l'auteur que par la précision et l'élégance de l'écriture. La lecture de ce gros volume n'est, en aucun moment, lassante et c'est l'un des grands 
mérites de Christine Laurière que de rendre vivante et attachante cette figure singulière de l'anthropologie française que fut Rivet.

2 Ce n'est pas un hasard si peu d'auteurs s'aventurent sur le terrain de la biographie intellectuelle; ce genre d'écrit requiert, de la part du biographe, de solides connaissances dans le domaine de l'histoire scientifique, sociale, institutionnelle et politique, tout comme une constante mise en perspective des documents d'archives avec les enjeux et les débats intellectuels d'une époque. De ce point de vue, le livre de Christine Laurière est une véritable biographie intellectuelle qui sait faire dialoguer les nombreux documents d'archives avec une solide maîtrise des textes de Rivet et de ses contemporains. Quand on sait les multiples centres d'intérêt du fondateur du musée de l'Homme, allant de la linguistique à l'anthropologie physique en passant par l'ethnologie et l'étude de la technologie, on ne peut que saluer le défi que constitue cette biographie d'un personnage aussi riche et complexe. De plus, c'est la vie intellectuelle et politique française dans l'entre-deux-guerres qui est retracée tout au long de ces pages.

3 L'ouvrage est divisé en quatre parties: la première est consacrée à la mission géodésique en Équateur (1901-1906) au cours de laquelle le jeune médecin militaire du service de Santé découvre sa vocation d'ethnologue. Dans la deuxième partie, Christine Laurière s'attache à rendre intelligible l'abandon progressif de la part de Rivet, à la suite de son retour de l'Équateur, de l'anthropologie physique au profit de la linguistique et de l'anthropologie diffusionniste. La troisième section est centrée sur les efforts institutionnels de Rivet en faveur du développement à la fois de l'américanisme et de l'ethnologie. La dernière partie du livre couvre les années 1933-1944 avec l'activité politique et militante de Rivet, sa lutte contre le fascisme et le racisme, la Résistance et l'exil en Colombie entre 1941 et 1943.

4 Il ne saurait être question, dans ce cadre limité, de rendre compte de l'activité savante et de l'engagement citoyen de l'anthropologue français. Si le rôle joué par Rivet dans la création de l'Institut d'ethnologie en 1925 et dans celle du musée de l'Homme est relativement bien connu grâce aux travaux de Jean Jamin et de Filippo Zerilli ${ }^{1}$, on connaît moins bien en revanche ses activités scientifiques et institutionnelles au début $\mathrm{du} \mathrm{xx}^{\mathrm{e}}$ siècle et pendant les années d'exil en Amérique latine. Ainsi, l'un des grands apports de ce livre réside dans l'analyse minutieuse des positions théoriques de Rivet qui, tout en prônant une approche diffusionniste, a cependant refusé les reconstructions de l'école historico-culturelle viennoise. Défenseur de la méthode cartographique et statistique, ce savant a évité ainsi de prendre parti en faveur du point de vue historique (diffusionniste) et du point de vue évolutionniste. C'est par la linguistique que Rivet est amené à examiner la question des origines de l'homme américain; en suggérant un apparentement entre les populations océaniennes et américaines, il s'opposait à l'anthropologue physique Ales Hrdlicka, du Bureau of Ethnology de Washington, et au dogme de l'origine exclusivement asiatique du peuplement du Nouveau Monde. Dans son analyse du débat sur les origines de l'homme américain, Christine Laurière souligne à juste titre la place qu'a occupée Rivet sur la scène scientifique internationale et la singularité de son approche, qui répertorie un vaste ensemble de traits culturels et technologiques, aspect quelque peu oublié dans l'histoire de l'anthropologie française.

5 On retiendra également de ce livre l'étude fine et détaillée de la mission de l'île de Pâques (1932-1935) entreprise par Henri Lavachery et Alfred Métraux. Dans les longues 
pages consacrées à cette mission, Christine Laurière fait état des doutes de Métraux au sujet de son expérience de terrain, de l'absence de coopération de la part des informateurs qui, imbus des théories de John MacMillan Brown, « les récitaient comme des légendes et traditions "connues de leurs ancêtres" " (p. 463), et de sa remise en question de l'hypothèse d'une parenté entre les civilisations américaines et océaniennes. Malgré le succès de l'exposition consacrée à l'île de Pâques tenue au musée du Trocadéro en 1935, dont la commissaire fut Divonne Ratton (l'épouse du célèbre marchand d'art Charles Ratton), les divergences théoriques entre Métraux et Rivet n'ont fait que se creuser. Relégué au Bishop Museum (Honolulu) à partir de 1936, Métraux s'est attaché à démontrer le caractère essentiellement polynésien de l'île de Pâques dans les colonnes de la revue de William Schmidt, Anthropos (1938), et par conséquent à réfuter la théorie (chère à Rivet) du double peuplement de cette île.

Parmi les nombreuses relations intellectuelles qui ont ponctué la longue vie de Rivet, c'est certainement avec Franz Boas que l'échange a été le plus fécond, tant pour ce qui est des affinités électives qu'en ce qui concerne l'engagement militant. Les deux chercheurs ne se sont connus personnellement qu'en 1924, après de longues années de correspondance, de soutien moral et aussi financier de la part de l'anthropologue nordaméricain au Journal de la Société des américanistes au lendemain de la Première Guerre mondiale. Savant et politique, Rivet ne pouvait qu'être en accord avec Boas sur la défense des valeurs de liberté, d'égale dignité des cultures et de justice, ce dont témoignent les quelques lettres émouvantes publiées récemment par Christine Laurière $^{2}$. Il importe de rappeler la façon dont Rivet a été «l'un des premiers anthropologues à remettre en cause les préjugés nourris envers les sociétés amazoniennes - immobiles, stagnantes, restées à l'écart des civilisations andines - et à briser la séparation idéologique entre les Indiens des hautes terres et ceux des basses terres » (p. 265).

7 Président du Comité de vigilance des intellectuels antifascistes, élu du Front populaire à Paris en 1935, député SFIO, Rivet fut un partisan de l'Algérie française, ce qui lui valut en 1949 l'exclusion du parti socialiste pour indiscipline de vote. Par ailleurs, et ce n'est pas l'un des moindres traits de cette personnalité insaisissable, il fut l'un des premiers à s'insurger contre la politique de répression et de torture menée par l'État français à Madagascar.

8 L'originalité de cet ouvrage tient aussi à la place accordée aux illustrations; très nombreuses (plus de 170) et de diverse nature (lettres personnelles, photographies de Rivet et de ses missions, photographies anthropométriques prises en Équateur), les images, parce qu'elles sont insérées dans le corps du texte, établissent un dialogue fructueux avec l'argument du livre. Un index, une chronologie et une bibliographie de Rivet complètent ce livre qui restera une référence incontournable pour tous ceux qui s'intéressent à l'histoire de l'anthropologie en particulier et à l'histoire sociale, politique et intellectuelle française de l'entre-deux-guerres en général. 


\section{NOTES}

1. Parmi les nombreux écrits de Jean Jamin voir, entre autres, «Le savant et le politique : Paul Rivet (1876-1958) », Bulletins et Mémoires de la Société d'anthropologie de Paris, nouvelle série 1(3-4), 1989 : 277-294 ; Filippo Zerilli, Il lato oscuro dell'etnologia. Rome, CISU, 1998.

2. Voir Christine Laurière, «Franz Boas and Paul Rivet's relationship: militancy as a scientific commitment », History of Anthropology Newsletter 36(1), 2009: 10-21.

\section{AUTEUR}

\section{NÉLIA DIAS}

Nelia.Dias@iscte.pt 